\title{
The Vibrio campbellii quorum sensing signals have a different impact on virulence of the bacterium towards different crustacean hosts.
}

\begin{abstract}
Pathogenic bacteria communicate with small signal molecules in a process called quorum sensing, and they often use different signal molecules to regulate virulence gene expression. Vibrio campbellii, one of the major pathogens of aquatic organisms, regulates virulence gene expression by a three channel quorum sensing system. Here we show that although they use a common signal transduction cascade, the signal molecules have a different impact on the virulence of the bacterium towards different hosts, i.e. the brine shrimp Artemia franciscana and the commercially important giant freshwater prawn Macrobrachium rosenbergii. These results suggest that the use of multiple types of signal molecules to regulate virulence gene expression is one of the features that allow bacteria to infect different hosts. Our findings emphasize that it is highly important to study the efficacy of quorum sensing inhibitors as novel biocontrol agents under conditions that are as close as possible to the clinical situation.
\end{abstract}

Keyword: Quorum sensing; Vibriosis; Host-microbe interaction; Host-pathogen interaction. 